\title{
Papers
}

\section{Selective serotonin reuptake inhibitors (SSRIs) and suicide in adults: meta-analysis of drug company data from placebo controlled, randomised controlled trials submitted to the MHRA's safety review}

\author{
David Gunnell, Julia Saperia, Deborah Ashby
}

\begin{abstract}
Objective To investigate whether selective serotonin reuptake inhibitor (SSRI) antidepressants are associated with an increased risk of suicide related outcomes in adults. Design Meta-analysis of randomised controlled trials of SSRIs compared with placebo in adults submitted by pharmaceutical companies to the safety review of the Medicines and Healthcare products Regulatory Agency (MHRA).

Participants Over 40000 individuals participating in 477 randomised controlled trials.

Main outcome measures Suicide, non-fatal self harm, and suicidal thoughts.

Results An estimated 16 suicides, 172 episodes of non-fatal self harm, and 177 episodes of suicidal thoughts were reported. We found no evidence that SSRIs increased the risk of suicide, but important protective or hazardous effects cannot be excluded (odds ratio $0.85,95 \%$ credible interval 0.20 to 3.40 ). We found weak evidence of an increased risk of self harm (1.57, 0.99 to 2.55). Risk estimates for suicidal thoughts were compatible with a modest protective or adverse effect $(0.77,0.37$ to 1.55$)$. The relative frequency of reported self harm and suicidal thoughts in the trials compared with suicide indicates non-fatal end points were under-recorded.

Conclusion Increased risks of suicide and self harm caused by SSRIs cannot be ruled out, but larger trials with longer follow up are required to assess the balance of risks and benefits fully. Any such risks should be balanced against the effectiveness of SSRIs in treating depression. When prescribing SSRIs, clinicians should warn patients of the possible risk of suicidal behaviour and monitor patients closely in the early stages of treatment.
\end{abstract}

\section{Introduction}

Depression affects around one in 38 adults in Britain at any point in time. ${ }^{1}$ The most serious and distressing consequence of depression is suicide, and concerns have arisen that the very drugs used to treat depression, and by implication reduce the risk of suicide, may in some susceptible individuals cause suicidal feelings, self harm, and suicide. ${ }^{2345}$ This concern is borne out by recent reviews of evidence from randomised controlled trials of selective serotonin reuptake inhibitors (SSRIs) compared with placebo in children. ${ }^{6}{ }^{2}$ However, it is uncertain whether such a risk is present in adults and whether risks are increased for suicide as well as non-fatal suicidal end points. A meta-analysis of data for fluoxetine (an SSRI), funded by its manufacturer, found no evidence that suicidal acts were more common among adults receiving active treatment, but the review lacked power $(\mathrm{n}=32$ episodes of suicide and non-fatal self harm) to identify important risks. $^{7}$ Khan et al synthesised clinical trial data for nine antidepressants and found, if anything, that suicide rates in people treated with placebo were lower than in those taking SSRIs or other antidepressants. ${ }^{8}$ Their findings are difficult to interpret as they had not conducted a formal meta-analysis.

We used data from the review of the safety of SSRIs that was recently published by the Medicine and Healthcare products Regulatory Agency (MHRA) ${ }^{9}$ to carry out a meta-analysis of data from placebo controlled, randomised controlled trials in adults to assess whether adults prescribed the SSRIs have an increased risk of suicide, non-fatal self harm, or suicidal thoughts.

\section{Methods}

\section{Data sources}

We abstracted data on the number of suicides, episodes of nonfatal self harm, and suicidal thoughts reported in placebo controlled trials of SSRIs in adults from the MHRA's review of the safety of SSRIs. ${ }^{9}$ For each SSRI (citalopram, escitalopram, fluoxetine, fluvoxamine, paroxetine, and sertraline), the pharmaceutical companies provided the MHRA with summed end point data across all trials, for all indications, separately in subjects treated with placebo and with the intervention. We did not have access to individual patients' data. Most trials were carried out to assess the effectiveness of drugs in treating depression, but the data summarised in the MHRA report ${ }^{9}$ included trials for other indications (such as obsessive compulsive disorder, anxiety, etc) and it was not possible to examine indication specific associations with risk. The exception to this was citalopram, where the presented data were only for trials of its use in depression rather than across a broader range of indications.

The manufacturers supplied data for each product from 477 trials, ranging from nine trials for citalopram to 156 for sertraline. 9 Data on the mean duration of follow up in the placebo and active arms of the trials were not available for all products, and so we were unable to take account of such possible differences in our analysis. Separate data for suicide and non-fatal self harm were not available for fluoxetine, and so the data for suicidal behaviour in relation to fluoxetine are presented in the analysis of non-fatal self harm as most of the events were likely to be non-fatal. Data for suicidal thoughts and non-fatal self harm were not presented separately for paroxetine. We therefore excluded the paroxetine data from the main synthesis for non-fatal self harm and suicidal thoughts, but we carried out 
Summary of clinical trial data abstracted from the Medicine and Healthcare products Regulatory Agency's review of the safety of SSRIs ${ }^{9}$

\begin{tabular}{|c|c|c|c|c|}
\hline \multirow{2}{*}{$\begin{array}{l}\text { SSRI (conditions included in RCTs; No of trials } \\
\text { contributing data) }\end{array}$} & \multicolumn{2}{|c|}{ Active (SSRI) arm } & \multicolumn{2}{|c|}{ Placebo arm } \\
\hline & No of subjects & No of episodes & No of subjects & No of episodes \\
\hline \multicolumn{5}{|l|}{ (a) Suicides in placebo controlled trials in adults } \\
\hline Citalopram (depression; 9 trials) & 1320 & 1 & 622 & 1 \\
\hline Escitalopram (all indications; 34 trials) & 2648 & $1^{*}$ & 2088 & 1 \\
\hline \multicolumn{5}{|l|}{ Fluoxetine† } \\
\hline Fluvoxamine (all indications; 48 trials) & 4186 & 2 & 3396 & 2 \\
\hline Paroxetine (all indications; 95 trials) & 8481 & 1 & 5808 & $3 \ddagger$ \\
\hline Sertraline (all indications; 156 trials) & 7169 & 4 & 5108 & 0 \\
\hline Total & 23804 & 9 & 17022 & 7 \\
\hline
\end{tabular}

Pooled odds ratio from bayesian random effects meta-analysis: 0.85 (95\% credible interval 0.20 to 3.40 )

(b) Non-fatal self harm in placebo controlled trials in adults

\begin{tabular}{|c|c|c|c|c|}
\hline Citalopram (depression; 9 trials) & 1320 & 11 & 622 & 5 \\
\hline Escitalopram (all indications; 34 trials) & 2648 & 6 & 2088 & 1 \\
\hline Fluoxetine (all indications; 135 trials)§ & 7010 & 17 & 4667 & 11 \\
\hline Fluvoxamine (all indications; 48 trials) & 4186 & 24 & 3396 & 10 \\
\hline Paroxetine (all indications; 95 trials)ף & 8481 & 33 & 5808 & 26 \\
\hline Sertraline (all indications; 156 trials) & 7169 & 20 & 5108 & 8 \\
\hline Total & 30814 & 78 (+ 33 paroxetine) & 21689 & 35 (+26 paroxetine) \\
\hline
\end{tabular}

Pooled odds ratio from bayesian random effects meta-analysis: 1.57 (95\% credible interval 0.99 to $2.55 ; 1.29,0.90$ to 1.91 , with paroxetine data included)

(c) Suicidal thoughts in placebo controlled trials in adults

\begin{tabular}{|c|c|c|c|c|}
\hline Citalopram (depression; 9 trials) & 1320 & 10 & 622 & 4 \\
\hline Escitalopram (all indications; 34 trials) & 2648 & 1 & 2088 & 2 \\
\hline Fluoxetine (all indications; 135 trials) & 3078 & 24 & 1800 & 31 \\
\hline Fluvoxamine (all indications; 48 trials) & 4186 & 23 & 3396 & 12 \\
\hline Paroxetine (all indications; (95 trials)ף & 8481 & 32 & 5808 & 26 \\
\hline Sertraline (all indications; 156 trials) & 7169 & 6 & 5108 & 6 \\
\hline Total & 26882 & 64 (+32 paroxetine) & 18822 & 55 (+26 paroxetine) \\
\hline
\end{tabular}

Pooled odds ratio from bayesian random effects meta-analysis: 0.77 (credible interval 0.37 to $1.55 ; 0.79,0.48$ to 1.28 , with paroxetine data included)

* Suicide occurred six days after stopping treatment.

†Data on suicide and self harm were not reported separately for fluoxetine.

$\ddagger$ All three placebo suicides occurred in the post-treatment period.

§Data on suicide and self harm were not reported separately for fluoxetine, so a few events listed here may be suicides.

IData for non-fatal self harm and suicidal thoughts not reported separately for paroxetine, so events assumed to be evenly distributed across these two outcomes.

a sensitivity analysis including the data and assuming half the events in each arm of the pooled trial data were non-fatal self harm and half were suicidal thoughts. As the number of events in the active arm of the paroxetine trials was 65 , which is not divisible exactly by two, we allocated 33 events to the self harm outcome and 32 to suicidal thoughts.

\section{Meta-analysis}

We used a bayesian random effects model ${ }^{10}{ }^{11}$ to synthesise data across the different SSRIs for each of the three outcomes. The priors for all parameters were vague (normal $\left(0,10^{-6}\right)$ for the mean of the log odds ratio, gamma $(0.001,0.001)$ for the precision of the means, and gamma $\left(10^{-4}, 10^{-4}\right)$ for the between products precision except for suicide data where we used gamma $(1,1)$ to ensure convergence). We present $95 \%$ credible intervals (the bayesian equivalent of confidence intervals) for the effect estimates.

We assessed heterogeneity in drug effects using the $\mathrm{I}^{2}$ statistic, which measures the proportion of variability owing to variation between products. ${ }^{12}$ We used Stata software version 8.0 (Texas, StataCorp, 2003) to produce figures and give the corresponding classical random effects estimates, for comparison. In our metaanalysis of the suicide data we used a continuity correction of 0.5 to overcome problems caused by small numbers. Using a continuity correction of 0.1 gave similar results.

\section{Sample size calculations}

We estimated sample size requirements for clinical trials to detect risks of self harm and suicide in relation to SSRI prescribing, based on the assumption of $80 \%$ power and 5\% level of significance using Stata.

\section{Results}

The MHRA's report presents summary data from 342 placebo controlled trials of SSRIs including 40826 subjects and 16 suicides. An estimated 172 episodes of non-fatal self harm were reported in data from 477 trials including 52503 subjects. For suicidal thoughts, data are presented from 477 trials of 45704 subjects and an estimated 177 episodes (table).

The pooled odds ratio (with 95\% credible intervals) for all SSRIs compared with placebo treated subjects in relation to suicide was 0.85 (0.20 to 3.40). For non-fatal self harm, excluding paroxetine, the odds ratio was 1.57 (0.99 to 2.55), and for suicidal thoughts, excluding paroxetine, it was 0.77 (0.37 to 1.55$)$. The $\mathrm{I}^{2}$ values were $27 \%, 3 \%$, and $37 \%$, respectively, indicating relatively little heterogeneity across the individual drugs for the first two outcomes. Three suicides in placebo treated patients (all in paroxetine trials) and one suicide among people treated with an SSRI (escitalopram) occurred after they had stopped treatment. Exclusion of these events from our meta-analysis resulted in a revised odds ratio for suicide of 1.24 (0.21 to 6.71). When we included paroxetine data for non-fatal self harm and suicidal thoughts in the meta-analysis, assuming half the events in each arm of the trial were non-fatal self harm and half were suicidal thoughts, the respective odds ratios were 1.29 (0.90 to 1.91) and 0.79 ( 0.48 to 1.28$)$. These odds ratios may be biased towards null effects as our assumption of an even distribution of episodes of self harm and suicidal thoughts across placebo and control groups is not supported by our meta-analysis of these end points excluding paroxetine data. 


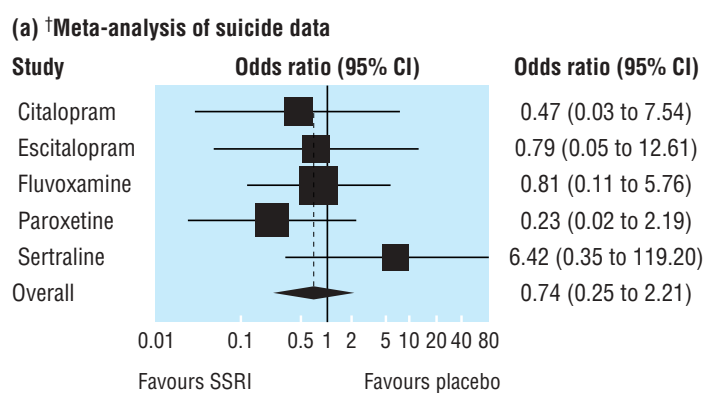

(b) Meta-analysis of non-fatal self harm data (excluding and including paroxetine)

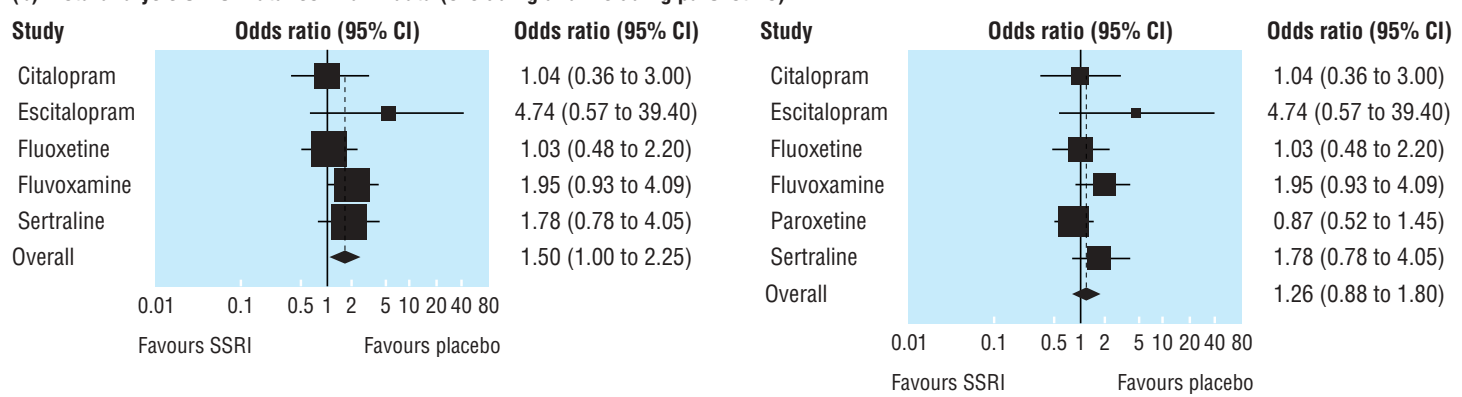

(c) Meta-analysis of suicidal thoughts data (excluding and including paroxetine)

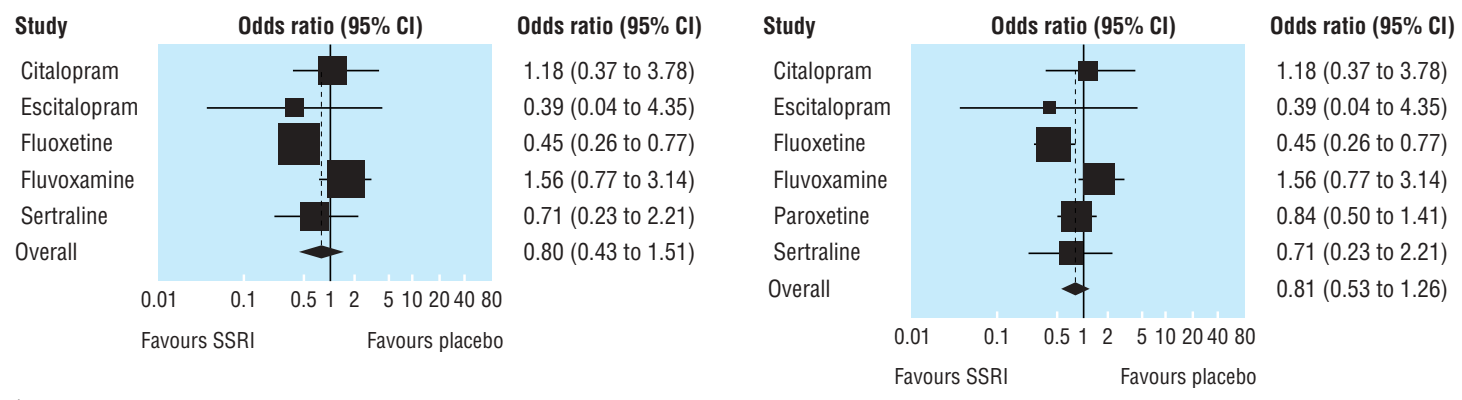

$\dagger$ With continuity correction of 0.5

Forest plots of suicide, non-fatal self harm, and suicidal thoughts in placebo controlled trials of SSRIs

All 95\% credible intervals are compatible with no increase in risk. The $95 \%$ credible interval for suicide is wide because of the small number of events. However, while the credible intervals for the risk of non-fatal self harm are also compatible with at least a doubling of risk and little evidence of risk reduction, those for suicidal thoughts are compatible with up to a two thirds reduction or a modest increase in risk. For non-fatal self harm, the number needed to treat to harm, using the odds ratio without paroxetine, is 759 (based on the weighted prevalence of self harm in the placebo groups of 1 in 433). As the $95 \%$ credible intervals for the odds ratio for non-fatal self harm span 1.0 they are compatible with both harm and benefit. Following Altman's suggestion, ${ }^{13}$ the $95 \%$ credible intervals around the numbers needed to treat to harm are $759(95 \%$ credible interval number needed to treat to harm 279 to $\infty$ to number needed to treat to benefit 43 300).

The figure shows the risk estimates for each SSRI in relation to suicide (a), non-fatal self harm (b), and suicidal thoughts (c). Because of the small number of suicides, the confidence intervals for the risk estimates are very wide. Of note, the Bayesian intervals are slightly wider than those in the figures, which have been calculated by using a classical approach, as the bayesian estimates reflect uncertainty about the precision between products.

The overall risk of suicide in both arms of the trials combined was 39 per 100000 (16 suicides among 40826 subjects). The risk of non-fatal self harm was about 10 times higher than that for suicide (328/100 000 (172 episodes of self harm among 52503 subjects)); the risk of suicidal thoughts was similar to that for non-fatal self harm (387/100000 (177 episodes of suicidal thoughts among 45704 subjects)). As the mean duration of the trials included in the synthesis was eight to 10 weeks, ${ }^{9}$ the overall rates of suicidal behaviour and thoughts per person year at risk are likely to be some five times higher than the risks calculated here.

\section{Size of trials needed to detect impact of SSRIs on risk of suicide and non-fatal self harm}

We based our sample size estimates on the risk of suicide $(39 / 100000)$ and non-fatal self harm (328/100 000) among those taking part in the randomised trials of SSRIs, assuming a $20 \%$ decrease in risk is considered clinically important. About 1.9 million subjects would need to be recruited to a trial to detect a $20 \%$ decrease in suicide risk (assuming $80 \%$ power and $5 \%$ level of significance). For non-fatal self harm, the total sample size would need to be about 220000 . To detect a halving of risk of suicide and self harm, the sample sizes required would be 262000 and 31000 respectively. 


\section{Discussion}

Because of the low incidence of suicide, it is not possible to rule out either a threefold increase or a decrease in its occurrence among people treated with SSRIs. Large trials, randomising around 2 million individuals, would be required to detect an important effect on risk. We found weak evidence of an increased risk of non-fatal self harm, but our results are compatible with either no reduction or a risk that is 2.5 times higher than in placebo treated patients.

The absence of evidence of an increased risk of suicidal thoughts, but possible increased risk of non-fatal self harm in relation to use of SSRIs, is noteworthy. These could be chance differences owing to the relatively small number of events. Alternatively the differences may arise as a result of drug efficacy in treating depression, and the possible rise in self harm may result from a different mechanism such as a disinhibiting effect of SSRIs in the early stages of treatment. Of note, the ratio of reported suicides to episodes of self harm (about 1:10) is lower than the ratio in the general population. About 5000 suicides $^{14}$ and over 142000 episodes of non-fatal self harm ${ }^{15}$ occur each year in England and Wales, giving a ratio of roughly 1:30. As over-reporting of suicides is unlikely, the lower ratio in the trials in our meta-analysis is likely to reflect under-reporting of episodes of self harm. Likewise the ratio of suicidal thoughts to self harm in the general population is around $1: 5,{ }^{16}$ yet the ratio in the studies included in this meta-analysis is closer to 1:1, suggesting under-reporting of suicidal thoughts. If the underreporting is biased in favour of SSRIs this could account for the possible differences in risk in relation to self harm and suicidal thoughts.

\section{Strengths of the analysis}

As our meta-analysis is based on trial data supplied by the relevant pharmaceutical companies and extracted according to guidelines from the MHRA, ${ }^{9}$ it includes both published and unpublished data. Publication bias is unlikely to be a problem. Unlike previous investigators analysing data from paediatric randomised controlled trials ${ }^{2}{ }^{6}$ we were able to distinguish between suicide, non-fatal suicidal behaviour, and suicidal thoughts.

\section{Limitations of the analysis}

The main limitation of our meta-analysis is that even with the pooling of data from several hundred randomised controlled trials in which over 40000 patients were randomised, we did not have sufficient power to detect clinically important risks or benefits. Meta-analyses of sparse data can be unstable. ${ }^{10}{ }^{17}$ Secondly, as we did not have access to individual patient data we could not conduct a meta-analysis based on individual patients or trials. For this reason heterogeneity between trials and individual products is likely to have been masked. Because of this underestimation of between trial variance our credible intervals will be underestimates of true variability. Thirdly, by pooling across different SSRI products we have made the implicit assumption that any adverse or beneficial effects of SSRIs are similar for all the products investigated. Sparse data on a small number of products make assessment of heterogeneity difficult. ${ }^{10}$ Fourthly, some relevant trial data are likely to have been excluded from this analysis as we did not carry out a systematic review of the published literature. In particular we will have excluded trials carried out by independent researchers with no involvement with pharmaceutical companies. Such trials are likely to present a less favourable picture of drug side effects. ${ }^{18}$ Fifthly, as already discussed, there is suggestive evidence that non-fatal self harm and suicidal thoughts were under-recorded. Lastly, most of the randomised controlled trials were of short duration (less than 10 weeks), and so possible longer term beneficial effects of SSRIs on suicidal behaviour will not have been detected.

\section{Previous studies}

The magnitudes of the risks of suicide and non-fatal self harm among adults in this meta-analysis are consistent with the findings of our synthesis of placebo controlled trials of SSRIs in children and adolescents (odds ratio 1.66, 95\% credible interval 0.83 to 3.50$){ }^{2}$ In Beasley's meta-analysis, in keeping with the findings for all SSRIs in this paper, adults treated with paroxetine reported fewer suicidal thoughts than patients treated with placebo.

Several observational studies based on the UK's General Practice Research Database (GPRD) have compared risk of suicide and non-fatal self harm among patients receiving different antidepressants. ${ }^{9} 190$ These have not compared risks in treated and untreated patients. Ecological studies comparing time trends in antidepressant prescribing with population suicide rates in several different countries provide no consistent evidence that recent increases in prescribing have been associated with adverse trends in suicide. ${ }^{2}$

\section{Implications for public health and clinical practice}

Systematic reviews confirm that SSRIs are effective treatments for adult depression ${ }^{21}$ and better tolerated by patients than the other main class of antidepressant-the tricyclic antidepressants. ${ }^{22}$ However, our meta-analysis indicates that it is possible, in the early weeks of treatment, that SSRIs are associated with an increased risk of suicidal behaviour. It is therefore important to consider the risks and benefits of these drugs. Meta-analyses of placebo controlled trials of antidepressants in adults indicate that the number of patients who need to be treated with an SSRI (fluoxetine) to get one response (defined according to both self report and clinicians' reports of patients being much or very much improved) is four to seven (see tables 2 and 3 in the paper by Bech et $\mathrm{al}^{23}$ ). Conversely, our data for non-fatal self harm indicate that the best estimate of the number needed to treat to harm is 759 . The balance may be less favourable in people with mild depression, where the effectiveness of SSRIs is unclear. ${ }^{24}$

Of note, in the trials submitted to the MHRA's recent review where a particular SSRI was compared with another SSRI or a non-SSRI antidepressant, no clear evidence was found of differences in risk between different classes of antidepressants. ${ }^{9}$ This observation indicates that any increases in risk are likely to be common to all antidepressants, rather than being specific to SSRIs.

\section{Conclusion}

In view of the widespread prescribing of SSRIs and the possibility that they may increase the risk of suicidal behaviour in some individuals, research is urgently needed both to clarify appropriate indications for their use and to determine whether it is possible to identify people at risk of possible suicidal side effects. In the meantime it is important that patients starting treatment with these drugs, as well as their carers, are counselled about these possible side effects, so they may make informed choices and receive appropriate monitoring.

Acknowledgments: The authors thank Richard Martin for comments on the paper and Doug Altman and Keith Hawton for their helpful reviews of the paper.

Contributors: DG and DA conceived the idea for the paper. JS carried out the data analysis. DG initially drafted the paper, and all authors have contributed to further drafting. DG is the guarantor.

Funding: None. 


\section{What is already known on this topic}

Selective serotonin reuptake inhibitor (SSRI) antidepressants are effective treatments for depression and other psychological disorders in adults

Recent evidence from randomised controlled trials indicates that SSRIs may increase the risk of self harm and suicidal thoughts in children and adolescents

\section{What this study adds}

Combined evidence from placebo controlled RCTs of SSRIs in adults cannot rule out either an important beneficial or harmful effect of SSRIs on suicide deaths

There is some evidence of an increased risk of non-fatal self harm in adults treated with SSRIs but no evidence of an increased risk of suicidal thoughts

The duration of most trials included in this review is too short to assess whether short term risks are offset by longer term benefits

Competing interests: DG and DA were members of the MHRA's expert working group on the safety of SSRIs. They acted as independent advisers, receiving travel expenses and a small fee for meeting attendance and reading materials in preparation for the meeting. DA has spoken on the methodology of adverse drugs reactions in HIV at a scientific meeting attended by several pharmaceutical companies, and sponsored by GlaxoSmithKline. An honorarium was paid to her department. JS has no competing interests.

1 Singleton N, Bumpstead R, O'Brien M, Lee A, Meltzer H, and Office for National Statistics. Psychiatric morbidity among adults living in private households, 2000. London: Statistics. Psychiatric morbidity among adults living in private households, 2000. London:
Stationery Office, 2001.

2 Gunnell D, Ashby D. Antidepressants and suicide: what is the balance of benefit and harm? BMJ 2004;329:34-8.

3 Healy D, Langmaak C, Savage M. Suicide in the course of the treatment of depression. J Psychopharmacol 1999;13:94-9.

4 Healy D. Lines of evidence on the risks of suicide with selective serotonin reuptake inhibitors. Psychother Psychosomat 2003;72:71-9.

5 Nutt D. Death and dependence: current controversies over the selective serotonin reuptake inhibitors.J Psychopharmacol 2003;17:355-64.

6 Whittington CJ, Kendall T, Fonagy P, Cottrell D, Cotgrove A, Boddington E. Selective serotonin reuptake inhibitors in childhood depression: systematic review of published versus unpublished data. Lancet 2004;363:1341-5.
7 Beasley CM Jr, Dornseif BE, Bosomworth JC, Sayler ME, Rampey AH Jr, Heiligenstein $\mathrm{JH}$, et al. Fluoxetine and suicide: a meta-analysis of controlled trials of treatment for depression. BMJ 1991;303:685-92

8 Khan A, Khan S, Kolts R, Brown WA. Suicide rates in clinical trials of SSRIs, other antidepressants, and placebo: analysis of FDA reports. Am J Psychiatry 2003;160:790-2.

9 Report of the CSM Expert Working Group on the safety of selective serotonin reuptake inhibitors. 2004. www.mhra.gov.uk/news/2004/SSRIfinal.pdf (accessed 28 Jan 2005).

10 Spiegelhalter DJ, Abrams KR, Myles JP. Bayesian approaches to clinical trials and health-care evaluation. Chichester: Wiley, 2003.

11 Spiegelhalter DJ, Thomas A, Best NG. WinBUGS version 1.2 user manual. Cambridge: MRC Biostatistics Unit, 1999

12 Higgins JP, Thompson SG, Deeks JJ, Altman DG. Measuring inconsistency in meta-analyses. BMJ 2003;327:557-60.

13 Altman DG. Confidence intervals for the number needed to treat. BMJ 1998;317: 1309-12.

14 Kelly S, Bunting J. Trends in suicide in England and Wales, 1982-96. Population Trends 1998;92:29-41.

15 Hawton K, Fagg J, Simkin S, Bale E, Bond A. Trends in deliberate self-harm in Oxford, 1985-1995. Implications for clinical services and the prevention of suicide. Br J Psychiatry 1997;171:556-60.

16 Gunnell D, Harbord R, Singleton N, Jenkins R, Lewis G. Factors influencing the development and amelioration of suicidal thoughts in the general population. Cohort study. BrJ Psychiatry 2004;185:385-93.

17 Sweeting MJ, Sutton AJ, Lambert PC. What to add to nothing? Use and avoidance of continuity corrections in meta-analysis of sparse data. Stat Med 2004;23:1351-75.

18 Als-Nielsen B, Chen W, Gluud C, Kjaergard LL. Association of funding and conclusions in randomized drug trials. JAMA 2003;290:921-6.

19 Jick SS, Dean AD, Jick H. Antidepressants and suicide. BMJ 1995;310:215-8.

20 Jick H, Kaye JA, Jick SS. Antidepressants and the risk of suicidal behaviors. JAMA 2004;292:338-43.

21 Freemantle N, Long A, Mason J, Sheldon T, Song F, Watson P, et al. Effective health care: the treatment of depression in primary care. Leeds: University of Leeds, Department of Health, 1993.

22 MacGillivray S, Arroll B, Hatcher S, Ogston S, Reid I, Sullivan F, et al. Efficacy and tolerability of selective serotonin reuptake inhibitors compared with tricyclic antidepressants in depression treated in primary care: systematic review and meta-analysis. BMJ 2003;326:1014-9.

23 Bech P, Cialdella P, Haugh MC, Birkett MA, Hours A, Boissel JP, et al. Meta-analysis of randomised controlled trials of fluoxetine v. placebo and tricyclic antidepressants in the short-term treatment of major depression. Br J Psychiatry 2000;176:421-8.

24 National Collaborating Centre for Mental Health. Depression: management of depression in primary and secondary care. London: National Institute for Clinical Excellence, 2004.

$$
\text { (Accepted 20 January 2005) }
$$

bmj.com 2005;330:385

Department of Social Medicine, University of Bristol, Bristol BS8 2PR David Gunnell professor of epidemiology

Wolfson Institute of Preventive Medicine, Queen Mary, University of London, London EC1M 6BQ

Julia Saperia research assistant

Deborah Ashby professor of medical statistics

Correspondence to: D Gunnell D.J.Gunnell@bristol.ac.uk 